\title{
A importância do dissilicato de lítio na odontologia moderna: revisão narrativa
}

\author{
The importance of lithium dissilicate in modern dentistry: narrative review
}

La importancia del litio disilizado en la odontología moderna: revisón narrativa

José Milton de Aquino e Silva Neto ${ }^{1 *}$, Kerolainy Alves Costa $^{1}$, Leandro Henrique Santos Barbosa ${ }^{1}$, Altamiro Júnio Paranhos Cesar de Medonça', Cibelle Teresa de Albuquerque Machado ${ }^{1}$, Michelle Leão Bittencourt Brandão Medeiros ${ }^{1}$, Tayguara Cerqueira Cavalcanti'.

\section{RESUMO}

Objetivo: Demonstrar a importância da indubitabilidade do dissilicato de lítio utilizada em procedimentos clínicos de prensagem isostática e o design da restauração para só assim fazer a sua fabricação restauradora (CAD/CAM) pelos cirurgiões dentistas. Revisão bibliográfica: Na atualidade a cerâmica de dissilicato de lítiomanuseada pelo Cirurgião Dentistas (CD) é um dos materiais que mais certifica os resultados benéficos ao paciente, no momento em que se menciona a realização de dentes artificiais, dos quais dispõem como resultados benéficos o equilíbrio da consolidação e estabilidade da coloração dentaria empregada, bastante resistente ao que se refere ao processo de degradação química e sua deterioração, acondicionando suas particularidades e qualidades em sua superfície, aprofundando-se em suas técnicas utilizadas. Considerações Finais: $O$ dissilicato de Lítio demonstra ser um material de instrumentação alterável, sendo apropriado para distintas ocorrências clínicas. O tratamento que é o mais indicado de acordo com as literaturas são os que envolvem o sistema CAD/CAM, por causa do ótimo resultado final, além do seu tempo de fabricação ser rápido e sua confiabilidade inquestionável.

Palavras-chave: Cerâmica, Compostos químicos, Próteses e implantes, Restaurações intracoronárias.

\section{ABSTRACT}

Objective: Demonstrate the importance of the indubitability of lithium disilicate used in clinical procedures of isostatic pressing and the design of the restoration to only make its manufacturing restoration (CAD/CAM) by dental w3eweurgeons. Literature review: Currently, the lithium disilicate ceramic handled by the Dental Surgeon (CD) is one of the materials that most certifies the beneficial results to the patient when mentioning the realization of artificial teeth, of which the balance of consolidation has as beneficial results and stability of the dental color used, quite resistant to the process of chemical degradation and its deterioration, conditioning its particularities and qualities on its surface, deepening in its techniques used. Final Considerations: Lithium disilicate proves to be a changeable instrumentation material, being appropriate for different clinical occurrences. The treatment that is the most indicated according to the literature is that involving the CAD/CAM system, because of the excellent final result, in addition to its fast manufacturing time and unquestionable reliability.

Keywords: Ceramics, Chemical compounds, Prostheses and implants, Inlays.

\section{RESUMEN}

Objetivo: Demostrar la importancia de la indubitabilidad del disilicato de litio utilizado en los procedimientos clínicos de prensado isostático y el diseño de la restauración para que solo su restauración de fabricación (CAD/CAM) sea realizada por cirujanos dentales. Revisión bibliográfica: Actualmente, lacerámica de disilicato de litio manejada por elcirujano dental (CD) es uno de losmateriales que más certifica los resultados beneficiosos para el paciente, al mencionar lacreación de dientesartificiales, de loscualesel equilíbrio de laconsolidacióntiene como resultados beneficiosos. Y la estabilidade del color dental utilizado, bastante resistente al proceso de degradación química y su deterioro, condicionando sus particularidades y cualidadesensusuperficie, profundizandoenlas técnicas utilizadas. Consideracionesfinales: El disilicato de litiodemuestra ser un material de instrumentación cambiante, siendo apropriado para diferentes casos clínicos. El tratamiento más indicado segúnla literatura es el que involucra el sistema CAD/CAM, debido al excelente resultado final, además de su rápido tiempo de fabricación y suindudablefiabilidad.

Palabras clave: Cerámica, Compuestos químicos, Prótesis e implantes, Incrustaciones.

${ }^{1}$ Centro Universitário CESMAC (CESMAC), Maceió - AL. *E-mail: Milton_neto_166@hotmail.com SUBMETIDO EM: 7/2020 


\section{INTRODUÇÃO}

O desenvolvimento de materiais dentários e a possibilidade de replicar a anatomia dentária permitiram a inserção de cerâmica pelos profissionais odontológicos. Sendo elas usados pela primeira vez no século 18 com o objetivo de obtenção de dentes artificiais como foi o caso das próteses totais (GOMES EA, et al.2008).

Em meados do final do século XVIII, com a descoberta do forno elétrico foi originalizado uma nova forma e método de processamento de cerâmica e, no início do século XIX, foramutilizados coroa de porcelana, com esse desenvolvimento a cerâmica foi introduzida no campo da odontologia protética. Desde esse tempo, existem mudanças em seus processos de fabricações, composição, desempenho, indicações e métodos de uso, fazendo com que elas fossem levadas à uma cerâmica dentária com alta estética e biocompatibilidade, porém em alguns casos ainda é fraca, especialmente ao que se refere a sua espessura muito fina (CALIXTO R e MASSING N, 2015).

Com a elevada busca ao que se refere a estética e a longa durabilidade ao processo de restauração foi levado ao uso extensivo de cerâmica para atender a esses requisitos. Para atender à demanda, os fabricantes visam constantemente melhorias das propriedades essenciais: o mecanismo, processo de adesão e sua estética, para melhorar o desempenho e em contrapartida simplificar a tecnologia. A cerâmica de vidro reforçada pelo dissilicato de lítio tem a capacidade e potencial de reproduzir a estética e a resistência similares às estruturas dos elementos dentais. Embora seu custo seja elevado por causa do processamento dessas cerâmicas, elas ainda dispõem de muitas propriedades vantajosas, como: resistência à fratura, estética suficiente, nenhuma estrutura de base metálica ou opaca e bom transluzimento (KALAVACHARLA VK, et al., 2015).

Hoje, a crescente demanda de padronização de elementos dentários que buscam beleza e harmonia ao sorriso fez com quer os CD's buscassem a utilização de materiais protéticos que exibam uma boa aparência mecânica para seus pacientes, podendo ser observado que esses materiais são cada vez mais utilizados em cerâmica. Dessa forma, pode-se observar que os componentes presentes na estrutura do dissilicato de lítio possuem uma forma inorgânica formada por oxigênio, que pode ter o princípio de agregação de alguns metais ou semi-metais (FERREIRA HA, et al., 2016).

O sistema cerâmico dispõe muitas propriedades com características boas, quando é utilizada como legatário dos dentes naturais, disponibiliza um bom coeficiente de expansão térmica, além de dispor de uma significativa estabilidade química, apresentando uma translucidez semelhante aos elementos dentais e é muito resistente ao desgaste e à pressão. Entretanto, para obtenção de resultados satisfatórios no padrão esperado, deve-se prestar atenção à integridade da aresta, caracterizando o formato da superfície, respeitando todas as estruturas anatômicas e, finalmente, considerar a tonalidade de cor que irá ser diferenciada de um paciente para outro (CALIXTO R e MASSING N, 2015).

O reparo em restaurações estabelecidas de forma indireta pode ser cimentado por técnicas com metodologias tradicionais de cimentação, sendo usado cimento de ionômero, os que são modificados por resina, cimento de fosfato de zinco ou ligação adesiva, e os CD's devem estar cientes de suas características, adaptação, desvantagens e vantagens, que são cruciais na hora da escolha (LEE HY, et al., 2017). Entre as cerâmicas à base de dissilicato de lítio, os elementos que constituem a resina são mais adequados devido à sua excelência estética e além de disponibilizar resistência à tração, flexibilidade, elevada resistência à fratura e baixo índice de processos infiltrativos nas bordas dos ângulos em comparação com outros adesivos. Além de ser bastante resistência aos fluidos orais. A ligação entre o adesivo de resina e a matriz dentária produz uma adesão forte e estável, garantindo o sucesso da restauração estabelecidas de formas indiretas (ANUSAVICE KJ, et al., 2013).

A cerâmica dentária classifica-se de acordo com a sensibilidade que sua superfície apresenta. Quando se refere as cerâmicas ácido-resistentes, a sua forma de condicionamento com o ácido fluorídrico apresenta pouca ou nenhuma degradação na superfície, porque o teor de óxido de silício é muito baixo ou nulo, assim como a zircônia. No grupo de cerâmica sensível ao ácido, o tratamento com ácido fluorídrico faz com que a matriz vítrea da cerâmica se degrade, promovendo a remoção seletiva da matriz vítrea, expondo a matriz 
cristalina, resultando na geração de microporos, que servirão como retentor micromecânico. São incluídas na constituição da cerâmica sensível a ácidos as constituintes de forma feldspata, feldspato reforçado por leucita e dissilicato de lítio (AMOROSO AP, et al., 2012).

Existem várias formas técnicas para o tratamento da superfície de procedimentos restauradores que envolvem as cerâmicas, dos quais pode-se dispor de: jateamento de óxido com alumina, jateamento com óxido de silício, condicionamento com ácido fluorídrico e uso de adesivos disponíveis no mercado (SHRIVASTAVA S, et al., 2014).

Nos dias que de hoje, um adesivo universal (MUA) também foi implantado, tendo como principal objetivo de simplificar a tecnologia na hora da sua aplicação, proporcionando versatilidade e reduzindo o tempo de manejo na hora do trabalho, já que esses produtos são disponibilizados em apenas um recipiente dos quais possuem em sua composição silano e um MDP (metacriloiloxidecil fosfato di-hidrogênio) dos quais ajudam de forma direta e auxiliadora com os agentes cimentantes (KALAVACHARLA VK, et al., 2015).

Na cerâmica de dissilicato de lítio, das quais são provenientes de grupos sensíveis ao ácido, o ácido hidrofluorídrico degenera a matriz vítrea, formando assim a parte mais superficial áspera da superfície interna da peça, adicionando desta forma uma maior forma de fixação da área. Devido às irregularidades geradas pelo ácido, o adesivo pode ser fixado à peça de trabalho, melhorando assim a retenção mecânica e o fortalecimento da resistência na hora de unir-se (BRUM R, et al., 2011). A cerâmica é semelhante à estrutura dos dentes, portanto pode ser bem adaptada aos tecidos periodontais e ter uma boa relação com os elementos que compõem o periodonto de proteção, ajudando a aumentar a vida útil do tratamento reparador (SHRIVASTAVA S, et al., 2014).

É importante prestar atenção em quando usar cada técnica, pois o processo de flexão e sua resistência são os sinais de escolha de uma cerâmica adequada em cada caso, podendo ser visto que na área dos dentes posteriores e dos elementos caninos, eles ganham maior resistência. A tensão requer cerâmica de alto rendimento com essas características (CALIXTO R e MASSING N, 2015). Dos inúmeros e mais diversos protocolos existentes para o processamento de superfícies cerâmicas, é importante destacar a possibilidade de combinar essas tecnologias de escolha de forma particularizada, sendo diferenciada de um paciente para outro e enfatizar a importância da compreensão profissional, além da habilidade dos mesmos para a reabilitação, pois cada material possui características próprias (BISPO LB, 2015).

Portanto, o principal objetivo deste trabalho é demonstrar a importância da indubitabilidade do dissilicato de lítio utilizada em procedimentos clínicos de prensagem isostática e o design da restauração para só assim fazer a sua fabricação restauradora (CAD/CAM) pelos Cirurgiões Dentistas.

\section{REVISÃO BIBLIOGRÁFICA}

Na última década, o número de materiais cerâmicos usados em restaurações totalmente cerâmicas aumentou e cada material tentou de maneiras diferentes melhorar suas propriedades mecânicas sem comprometer a estética. Para poder ocorrer a seleção de forma correta os materiais devem ser estabelecidos de acordo com cada caso de forma particularizada, buscando sempre respeitar e manter a estrutura do elemento dental analisando assim os requisitos fundamentais para a reabilitação de preservação através de manutenções, o desempenho e a estética do sorriso, também é necessário entender certas características desses materiais (HELVEY GA, 2014).

Embora seja difícil agrupar vários sistemas cerâmicos, existem várias maneiras de classificar esses materiais e alguns métodos de classificação são propostos (CALIXTO R e MASSING N, 2015). A classificação da cerâmica é designada conforme a sua constituição, metodologia aplicada no processamento, força ao que se refere a resistência, grau de temperatura de fusão, transparência, indicações clínicas (dentes anteriores ou posteriores, coroa parcial ou completa), cimentação (forma adesiva ou tradicional) e sensibilidade superficial das quais essas características peculiares podem simplificar a avaliação, seleção de material para cada situação encontrada (SHRIVASTAVA S, et al., 2014).

A cerâmica é um sólido inorgânico e não metálico, produzido por aquecimento ou resfriamento dos componentes em seu estado original, sendo adquiro individualmente ou misturados. As cerâmicas dentárias 
são derivadas de outras tecnologias ou possuem alguns componentes orgânicos em sua composição. Essas cerâmicas podem conter estruturas total ou parcialmente cristalinas ou podem ser amorfas, como o vidro. (MAGNO P e BELSER U, 2012).

São materiais usados para replicar próteses dentarias usadas para reparar dentes perdidos ou danificados e também são conhecidos como porcelana dentária. São materiais semelhantes aos dentes naturais, porque possuem propriedades ópticas, excelente estética e longevidade, e apresentam sinais de vida muito longos (HELVEY GA, 2014).

\section{Microestrutura da cerâmica dissilicato de lítio}

Entre as cerâmicas disponíveis, o dissilicato de lítio é o mais proeminente, pois são utilizados em vidro cerâmico e amplamente conhecido como um de seus principais representantes o IPS (IvokraVivadt). Esses materiais cerâmicos são bastante tolerantes ao choque térmico, por causa de sua baixa expansão térmica causada por seu processamento. Outra das suas qualidades é que o dissilicato de lítio é translúcido, após o material atingir a cor necessária, converte metassilicato de lítio em dissilicato de lítio pelo processo de condensação. Por outro lado, pode ser observado que é fornecido alta seleção de opacidade, o sistema cerâmico pode ser processado pela técnica de cera perdida, prensagem ou fresagem isostática a quente usando o sistema CAD / CAM (auxiliado por computador Design / Fabricação Assistida por Computador) (VECHIATO FILHO AJ, 2014).

Diante desse exposto as cerâmicas reforçadas com dissilicato de lítio (IPS Empress2®-IvoclarVivadent) apresentam boas particularidades ao que se refere as propriedades mecânicas e resistência, tendo uma resistência à compressão de 300 a 400 Mega Pascal (Mpa). Este material foi modificado e estabeleceu o sistema IPS O e.max (IvoclarVivadent) dos quais dispõe superiores características em sua propriedade de tração mecânica, especialmente por causa da minimização das suas partículas de cristais e a interligação das quais eles possuem melhorando assim o intertravamento entre eles, tornando-se mais compactos, bloqueando desta forma as possíveis propagações de trincas (Kang SH, et al., 2013).

As Cerâmica que possuem seus reforços através de dissilicato de lítio devido os seus resultados satisfatórios ao que se refere as propriedades estéticas, forma de biocompatibilidade com a estrutura dentária, boa adaptabilidade das bordas, maior resistência ao desgaste, estabilidade de coloração dos elementos dentais, acúmulo mínimo de placa e baixa condutividade térmica, é considerado um material de escolha e pode ser utilizado em grande escala para vários tipos de reparos, desde os indiretos, como facetas laminadas, coroas simples, até próteses fixas com até 3 elementos, inlays, onlays e overlays (COLARES RM, et al., 2013).

\section{Processo de adesão da cerâmica dissilicato de lítio}

Em reparos indiretos feitos em restaurações, a adesão efetiva e duradoura entre resina e cerâmica pode garantir uma maior resistência à possíveis fratura, podendo melhorar a retenção e reduzir a incidência de microinfiltração na hora do preparo. Essa combinação se estabelece pela retenção micromecânica e adesão de forma química na superfície da cerâmica à base de sílica. Quando a superfície da cerâmica é tratada com o ácido fluorídrico, são geradas micro-irregularidades na parte mais superficial através da degradação da matriz de vidro, obtendo assim a retenção micromecânica, produzidos pelo ácido fluorídrico que variam com a concentração do ácido e o tempo de ajuste proposto na hora do manuseio, resultando assim diversas e diferentes irregularidades e valores de resistência à flexão da cerâmica (PRADO M. et al., 2018).

Acompanhando os mesmos conceitos das resinas compostas tradicionais, as cerâmicas possuem em sua composição materiais compósitos, isto é, são estabelecidas a partir de vários constituintes, como é o caso do vidro presente em sua matriz, onde as partículas de vidro ou cristal penetram leve ou fortemente em altas temperaturas (MCLAREN EA e FIGUEIRA J, 2015).

O objetivo de um sistema completo em material cerâmico surgiu para eliminar a estrutura metalocerâmica (conjunto de estrutura metálica coberta com porcelana), ocasionando um excelente desempenho, possuindo boa estética devido à distribuição da reflexão da luz (MAGNO P e BELSER U, 2012). 
Com o objetivo de fazer com que as cerâmicas vítreas conseguissem ser aplicadas de forma segura em coroas totais posteriores ou próteses fixas, os seus compostos presentes em suas características particulares necessitavam ser aprimorados, com isso foi reforçado com dissilicato de Lítio no sistema IPS EMPRESS2, do qual foi possibilitado estabelecer grandefirmeza a fratura, além de possuir uma elevadaforçaem sua estrutura (AMOROSO AP, et al., 2017).

Essas cerâmicas ao longo dos anos vêm sendo cada vez mais utilizadas por conta da estética que proporciona ao paciente e sua confiabilidade, no entanto, para obter um reparo suficiente, o processo de consolidação de excelente qualidade na restauração é crucial, uma das intercorrências que possam vim à surgir são relacionadas as restaurações com falhas, sendo estes e os ajustamentos oclusais que irão estabelecer o resultado positivo ou negativo do processo restaurador (PRADO M. et al., 2018).

\section{Técnicas de processamento para cerâmicas de dissilicato de lítio}

São estabelecidos lingotes de cerâmica de dissilicato de lítio para o processamento através de prensagem com ar quente, buscando assim impedir o surgimento de defeitos como poros ou pigmentos na hora da fabricação. A microestrutura dessas pastilhas constitui-se por volta de $60 \%$ a $70 \%$ de constituintes de cristais de dissilicato dos quais são incorporados e ligados em uma matriz de vidro. A cor desta cerâmica é obtida através do dissolvimento dos íons polivalentes em suas matrizes, esse processo dependerá da valência do campo elétrico ao redor dos íons presentes, e esses íons controlarão a cor uniformemente na fase inicial do material, eliminando assim quaisquer defeitos na estrutura (BORGES G, et al., 2015).

As estruturas dos blocos de fresagem são adquiridos através de um processo semelhante aos blocos utilizados para prensagem. No entanto, eles irão passar por um procedimento chamado cristalização intermediária do qual é um local para garantir a excelência e eficiência do produto após a moagem (fresamento). Esta etapa é chamada Azul translúcido. O processo intermediário de cristalização produzirá cristais de metassilicato de lítio, dos quais irá garantir uma satisfatória qualidade em sua estabilidade e reduzirá rachaduras em áreas finas. Só depois dessas etapas o material a ser triturado e sintetizado atingi a forma de cristalização e firmeza (SILVA NJMA, et al., 2020).

Estes blocos geralmente são utilizados em CAD/CAM, a tecnologia foi utilizada desde o ano de 1970, podendo ser observado que cada vez mais tem havido maiores aplicabilidade no campo odontológico pelos Cirurgiões dentistas, esses fatores acontecem por causa da sua praticidade e diminuição do tempo no consultório melhorando por sua vez o desempenho do profissional e obtendo indicies de satisfações melhores dos pacientes. Três componentes são de suma importância na hora de fabricação, o scanner, software para o desenvolvimento do design digital das estruturas dentarias e anatomia e seu sistema de fresagem.

O scanner é usado para obter dados em 3D, convertendo-os em materiais digitais, essas informações podem ser obtidas das seguintes maneiras: Scanner intraoral ou por silicone de adição. Logo depois da obtenção da digitalização, o desenho é transmitido no software, onde a linha de acabamento, espaçamento e espessura a ser restaurada são definidas. O sistema de fresamento é responsável pelo processamento de parte do material, dos quais usa blocos pré-fabricados (MCLAREN EA e FIGUEIRA J, 2015).

Posteriormente, o processo de sinterização (metalurgia do pó), e caracterização é realizado em um forno específico, o CD deve possuir esse equipamento em sua clínica ou mandar para um laboratório terceirizado. Tendo o profissional que fazer um investimento à mais, pode ser observado no mercado que para $0 \mathrm{CD}$ conseguir uma aquisição de sistema CAD/CAM o custo é muito elevado. O CD também deve possuir as matérias com o objetivo de sobrepor o glaze para o esmalte para poder forneça as características e conhecimentos anatômicos corretos, caso contrário, na clínica do profissional deve possuir um profissional protético ou é necessário fazer tudo isso com a ajuda de um laboratório protético, para atender aos requisitos do material após a fabricação. No entanto, esse sistema é considerado versátil, todavia, além do CD possuir a necessidade do conhecimento teórico e prático correto das formas marginais de anatomização, da aplicação de cimentação, utilização do silano com finalidades de criação de camadas híbridas e fazer uma restauração correta. Desta forma pode-se concluir que o CD precisa possuir 
habilidades técnicas sobre dentistica e o sistema que irá trabalhar, nesse caso o CAD/CAM (FORNAINI C, et al., 2018).

A utilização desta técnica, deste modo consegue-se fazer a entregada coroa em meramente em uma consulta, assim sendo o paciente não carecerá de ir para outra consulta nem irá passar pelo incômodo de outro processo anestésico e não é imprescindível a elaboração de um elemento provisório (MCLAREN EA e FIGUEIRA J, 2015).

\section{Taxa de sucesso clínico da cerâmica de dissilicato de lítio}

Sabe-se que a utilização da cerâmica dissilicato de lítio realizada através de processamento de achatamento isostático disponibiliza maior força quando se comparada as que são produzidas com a tecnologia CAD/CAM, apesar disso, esta última técnica citada dispõe de fatores relacionados as pigmentações melhores do que a técnica de prensagem, uma das causas desses benefícios são por causa dos blocos de cerâmica que já estão pré-fabricados, o que diminui significativamente falhas indesejadas na hora da coloração.

No entanto, com as avançadas tecnológicas que se depara cada dia mais no mercado o sistema que utiliza a técnica CAD/CAM, propende a ser o tratamento elegível. Isso acontece por que os pacientes não querem passar muito tempo para obter o resultado final, e muitos não dispõem de tempo para ir em várias sessões clinicas, com isso esse sistema é o mais ideal, além de reduzir o tempo clinico do paciente, reduz a ansiedade do paciente (AMOROSO AP, et al., 2017).

Em suma, deve-se sempre levar em consideração que, além da escolha estabelecida de forma correta e do respeito ao plano de restauração, a reabilitação bem-sucedida com cerâmica principalmente as que são constituídas com dissilicato de lítio envolve vários aspectos dos quais tem que ser levado em consideração, como: preparação adequada dos dentes, adaptação das bordas marginais, respeito as angulações, anatomia dental, o processo de modelagem, interação com os tecidos moles, sabendo-se que resultados satisfatórios não dependem exclusivamente apenas da escolha correta do tipo de cerâmica utilizada, mas também da escolha adequada dos materiais utilizados, das técnicas aplicadas na hora da execução e habilidades dos CD's (SILVA NJMA, et al., 2020).

\section{CONSIDERAÇÕES FINAIS}

A revisão de literatura estabelecida consente entender-se que as cerâmicas que são estabelecidas com os princípios ativos de dissilicato de Lítio demonstram serem materiais de instrumentações alteráveis, sendo apropriados para distintas ocorrências clínicas, especialmente na ocasião em que o paciente está em busca de fatores estéticos. Essas cerâmicas são em sua totalidade confiáveis em relação a forma de degradação mecânica, química, biológica e dispõe de uma boa adaptação marginal, possibilitando facilidade na hora de ser feita a cimentação, formando um revestimento híbrido, observa-se que todos esses parâmetros são conseguidos contanto que bem estabelecidas em suas indicações e na hora da sua confecção. Entre suas vantagens pode-se destacar a ótima resistência, do qual estabelece garantia à restauração dispondo de bastante durabilidade. $O$ tratamento que é o mais indicado de acordo com as literaturas feitas nos dias de hoje são os que envolvem o sistema CAD/CAM, por causa do ótimo resultado final, além do seu tempo de fabricação ser rápido e sua confiabilidade inquestionável.

\section{REFERÊNCIAS}

1. AMOROSO AP, et al. Cerâmicas odontológicas: propriedades, indicações e considerações clínicas. Rev. Odontol. Araçatuba, Araçatuba, 2017; 33(2): 19-25.

2. ANUSAVICE KJ, et al. Cimentos dentários materiais dentários. 12. Rio de Janeiro. Ed. Saunders Elsevier. 2013; 14: 307-339.

3. BISPO LB. Cerâmicas odontológicas: vantagens e limitações da zircônia. Rev Bras.Odontol. 2015; 76(1⁄2): $24-29$. 
4. BORGES G, et al. Cerâmicas Odontológicas Restauradoras. Pro-Odonto Prótese e Dentística, 2015; 3: 9-64.

5. BRUM R, et al. The influence of surface standar dization oflithium dissilicate glasscera miconbonds trengthto a dual resincement. Oper Dent. 2011; 36-5: 478-485.

6. CALIXTO R, MASSING N. Longevidade das restaurações cerâmicas anteriores. Parte 1, Rev. Dental Press Estética, Araraquara, 2015; 18-28.

7. COLARES RM, et al. Effectof Surface Pretreat mentson the Microtensile Bond Strengthof Lithium-Disilicate Ceramic Repaired with Composite Resin. Braz. Dent. J., 2013; 24(4): 349-352.

8. FERREIRA HA, et al. influência de agentes clareadores nas propriedades superficiais (rugosidade e microdureza) de uma cerâmica odontológica, cerâmica. 2016: 55-59.

9. FORNAINI C, et al. Disilicate Dental Ceramic Surface Preparationby 1070nm Fiber Laser: Thermaland Ultrastructural Analysis. Bioengineering, 2018; 5(1): 1-11.

10. GOMES EA, et al. Ceramic in dentistry: currentsituation Cerâmica 54. 2008; 319 -325.

11. HELVEY GA. Classifying Dental Ceramics: Numerous Materials and Formulations Available for Indirect Restorations, Compendium. 2014; 38-43

12. KALAVACHARLA VK, et al. Influence of Etching Protocol and Silane Treatment with a Universal Adhesive on Lithium Disilicate Bond Strength. Oper Dent, 2015; 40(2): 01-07.

13. Kang $\mathrm{SH}$, et al. Flexuralstrengthandmicrostructureoftwolithiumdisilicateglassceramics for CAD/ CAM restoration in the dental clinic. Restor Dent Endod. 2013; 134-140.

14. LEE HY, et al. Bonding of the silane containing multi-mode universal adhesive for lithium disilicate ceramics. RDE, Seoul, 2017; 42(2): 95-104.

15. MAGNO P, BELSER U. Restaurações adesivas de porcelana na dentição anterior- Uma abordagem biomimética, $1^{\text {a }}$ ed. Quintessense Editora Ltda., São Paulo, 2012; 406.

16. MCLAREN EA, FIGUEIRA J. Updating classifications of ceramic dental materials: a guide to material selection. Inside Dentistry. 2015; 48-72.

17. PRADO M. et al. Ceramic surface treatment with a single-component primer: resina dhesion to glass ceramics. $J$ Adhes Dent, 2018; 20(02): 99-105.

18. RITTER RG. Multifunctional Uses of a Novel Ceramic-Lithium Disilicate. Wiley Periodicals, Estados Unidos, 2010; 22.

19. SHRIVASTAVA S, et al. Ceramic cementation: a key to succesfull restoration, Annals and Essences of Dentistry. Department of Prosthodontics, Crown Bridge and Implantology. India, 2014; 35-43.

20. SILVA NJMA, et al. O progresso das cerâmicas no uso odontológico: uma revisão de literatura. Revista Eletrônica Acervo Saúde, 2020; (42), e2753.

21. TRINDADE AC, et al. Quando dois se tornam um, Prótese News, 2016; 3(4): 394-399.

22. VECHIATO FILHO AJ. Análise da superfície de cerâmicas de dissilicato de lítio após imersão em soluções ácidas e a base de flúor. Dissertação (mestrado em Odontologia) - Serviço Técnico de Biblioteca e Documentação - FOA / UNESP, 2014 